\title{
Short- and long-term physiologic and pharmacologic control of blood pressure in pediatric patients
}

This article was published in the following Dove Press journal:

Integrated Blood Pressure Control

24 May 201I

Number of times this article has been viewed

\author{
Howard Trachtman \\ Division of Nephrology, Cohen \\ Children's Medical Center of \\ New York, Long Island Campus \\ for the Albert Einstein College of \\ Medicine, New Hyde Park, NY, USA
}

Correspondence: Howard Trachtman Cohen Children's Medical Center, Division of Nephrology, 269-0I 76th Avenue, New Hyde Park, NY I 1040, USA

Tel +I 7 I8 4703423

Fax + I 7184700887

Email htrachtm@nshs.edu
Abstract: The incidence of hypertension is rising in the general population. A parallel trend is present in children and adolescents. This reflects more intensive treatment and improved patient survival after a wide range of serious systemic illnesses that can lead to hypertension. In addition, primary or essential hypertension is more prevalent because of the epidemic of obesity and metabolic syndrome in pediatric and adult patients of both genders and in all ethnic groups. As a consequence of the changing demographic pattern of hypertension, more patients are requiring therapy for elevated blood pressure. This review summarizes key aspects of the treatment for hypertension in pediatric patients and the long-term management of this problem, including nonpharmacologic strategies and drug treatment.

Keywords: hypertension, pediatric, pharmacologic, renoprotection

\section{Introduction}

Early reports from the 1970s and early 1980s suggested that hypertension was an infrequent abnormality in pediatrics, affecting, at most, $3 \%-5 \%$ of children. This figure is consistent with early studies based on a single measurement of blood pressure and the statistical definition of hypertension as blood pressure exceeding the 95th percentile. Lower prevalence figures in the $1 \%-3 \%$ range were reported in studies that incorporated repeated measurements of blood pressure on at least three separate occasions. However, in recent years, there is growing documentation that hypertension is an increasingly common clinical problem in children and adolescents. ${ }^{1}$ This reflects the more widespread measurement of blood pressure as part of routine care in pediatric practice in conjunction with epidemiologic changes in dietary habits, exercise patterns, and obesity. In addition, more children are surviving with complex medical conditions that require treatment with agents that cause hypertension. As a consequence, there is an urgent need for general pediatricians and specialists to have a greater understanding of the etiology and management of hypertension in pediatric patients. This review summarizes current thinking about the causes of hypertension and outlines an approach to the short- and long-term therapy of hypertension. The treatment section will focus on the following clinical circumstances: (i) acute severe hypertension, (ii) chronic hypertension secondary to an underlying disease, and (iii) chronic asymptomatic primary hypertension.

\section{Causes of hypertension}

Hypertension in pediatric patients can either be primary or essential in nature or be secondary to a wide range of illnesses. The secondary causes can be categorized into 
five broad categories: (i) renal parenchymal disease, eg, acute and chronic glomerulonephritides, polycystic kidney disease, reflux nephropathy; (ii) renal vascular hypertension; (iii) aortic coarctation; (iv) endocrine disorders, eg, hyperthyroidism, hypercortisolism, hyperparathyroidism; (v) malignancies, eg, neuroblastoma, Wilms' tumor, pheochromocytoma; and (vi) miscellaneous disorders, eg, Guillain-Barré syndrome, immobilization, illicit drug use, administration of corticosteroids. It is worth noting that over the last 20 years, the vast majority of children and adolescents referred for evaluation in the Pediatric Hypertension Center at Cohen Children's Medical Center (New Hyde Park, NY) have essential hypertension. The most common secondary causes are renovascular disease and coarctation of the aorta. This reflects prior identification of children with kidney disease.

The cause of hypertension emerges from a comprehensive history and physical examination of the patient. Laboratory testing usually includes a comprehensive metabolic profile, a lipid profile, a renal ultrasound, and an echocardiogram. The yield of most of these routine diagnostic tests in patients with mild-to-moderate hypertension is low, with less than $10 \%$ revealing significant abnormalities. ${ }^{2}$ More specific tests, such as assays for plasma catecholamines and hormone levels and renal arteriography, should be guided by the specifics of the individual case. Measurement of blood pressure over a 24-hour period using portable ambulatory monitoring devices is widely used and is being incorporated into the standard evaluation of hypertension in children. ${ }^{3}$ Normative data are available for this procedure, and the test provides a very accurate assessment of blood pressure. For example, this test facilitates the discrimination between healthy children with normal blood pressure, those with masked hypertension (normal clinic blood pressure reading and high levels at home), white coat hypertension (high clinic blood pressure reading and normal levels at home), and sustained hypertension. In addition, it supplies useful information that cannot be ascertained by reliance solely on office measurements, including blood pressure load (percentage of readings above the 95th percentile, nocturnal dipping pattern, and mean pressure during specified periods of the day).

Hypertension has many causes and has important target organ consequences, such as left ventricular hypertrophy. Many subspecialists, including cardiologists, endocrinologists, and interventional radiologists, contribute to the successful evaluation and treatment of children with high blood pressure. However, the vast majority (over $80 \%$ of cases) of secondary causes of hypertension in children arise from primary kidney disease. ${ }^{4,5}$ Therefore, pediatric nephrologists have the greatest experience in the diagnosis and management of children with high blood pressure. It is for this reason that it is recommended that pediatric patients with newly identified hypertension should be referred to a pediatric nephrologist for their initial evaluation.

\section{Acute severe hypertension}

Blood pressure readings are considered severely elevated if they exceed the 99th percentile or if they are more than 20/10 $\mathrm{mm} \mathrm{Hg}$ above the 95th percentile level for the child's age, gender, and height. ${ }^{5,6}$ Blood pressure levels above the 99th percentile or more than four standard deviations (SDs) above the mean are considered severe, and there is a higher likelihood of symptoms and physical findings on examination. ${ }^{6}$ However, any high blood pressure in the presence of neurologic symptoms is an acute emergency that requires urgent therapy. The differential diagnosis is as broad as the list of causes of asymptomatic mild hypertension. However, in children, the commonest causes include acute glomerulonephritis, hemolytic uremic syndrome, reflux nephropathy, renal artery stenosis, and intoxications. Under these circumstances, there is a need for emergency treatment to lower blood pressure sufficiently to reduce the risk of vasogenic edema and cerebral hemorrhage. The target of treatment is not to normalize the blood pressure but rather to lower to the mean arterial pressure by $20 \%{ }^{7}$ Once this degree of blood pressure lowering is accomplished, the patient can be started on a regular regimen of medications designed to completely normalize the blood pressure readings. It is important to note one critical distinction between adult and pediatric patients with acute hypertensive crisis. In the former cohort, because of the presence of atherosclerosis and chronic vascular disease in the brain, heart, and gut, adequate organ perfusion may depend on maintenance of a relatively high perfusion pressure. Thus, acute lowering of blood pressure can cause vital organ ischemia and manifest as a myocardial infarction or cerebrovascular accident. Children are less likely to have atherosclerosis and therefore can tolerate sudden drastic drops in blood pressure. Although they may complain of symptoms such as lightheadedness, fatigue, palpitations, and nausea, they are highly unlikely to experience organ dysfunction. This makes the management of acute hypertensive emergencies much easier in pediatric patients.

In patients with severe hypertension $(>4$ SDs above the mean $)^{6}$ or those in whom the elevated blood pressure is accompanied by symptoms, it is advisable to initiate therapy with parenteral medications. The first-line agent used in 
pediatrics is labetalol. The dose is $0.25-1 \mathrm{mg} / \mathrm{kg}$ per dose administered by rapid infusion. The blood pressure response occurs within 5-10 minutes. The dose can be escalated to the maximal level or repeated in 30-60 minutes, depending on the adequacy of the response. Labetalol can also be provided as a continuous infusion, but this requires some time to prepare the solution and can be implemented after bolus injections of the drug have lowered the blood pressure to safer levels. A second choice is a calcium channel blocker (CCB), such as isradipine ( 0.1 dose $\mathrm{mg} / \mathrm{kg}$ per dose $)$ or nicardipine $(0.1-0.3 \mu \mathrm{g} / \mathrm{kg}$ per minute). These agents, which have a peak hypotensive within 2.5 hours, are generally effective regardless of the patient's age or the etiology of the hypertension. ${ }^{8}$ It should be noted that short-acting nifedipine has also been demonstrated to be safe and effective in hospitalized patients with severe acute hypertensive emergencies. ${ }^{9}$ However, its use has been curtailed because of reports of cardiovascular complications that occurred in adult patients who were given the drug for the control of life-threatening hypertension. A third option is enaliprilat, an injectable angiotensin-converting enzyme inhibitor (ACEI), provided in a dose of 0.005-0.1 $\mathrm{mg} / \mathrm{kg}$ per hour. Diazoxide, an agent that was widely used in the past, either as a single rapid infusion of $5 \mathrm{mg} / \mathrm{kg}$ or multiple small injections of $1 \mathrm{mg} / \mathrm{kg}$ per dose, has fallen out of favor because it can cause hyperglycemia and fluid retention. Moreover, in contrast to the other options, diazoxide is more likely to cause overshoot of the target blood pressure. Although hydralazine, in a dosage of $0.15-0.25 \mathrm{mg} / \mathrm{kg}$ every 4-6 hours, is highly effective, it is associated with severe reflex tachycardia and nausea, and it is less well tolerated compared with labetalol, a CCB, or an ACEI.

If the patient is awake and fully conscious, then severe hypertension can be controlled with a number of oral medications. These include enalapril, clonidine, and minoxidil. It is worth mentioning that short-acting nifedipine $(0.3 \mathrm{mg} / \mathrm{kg}$ per dose every $4-8$ hours $)$ remains a useful agent in this context. If the capsule is punctured, then the contents of the capsule are rapidly absorbed and the blood pressure-lowering action is manifest within 20-30 minutes. There is no sublingual absorption of the drug and there is no pharmacodynamic rationale to administer the drug in this fashion. Studies have confirmed the complete absence of absorption of nifedipine across the buccal mucosa and have verified that the antihypertensive action is related to the amount of the drug that is swallowed and absorbed from the stomach. ${ }^{10}$ In addition, it is important to emphasize that short-acting nifedipine is safe and effective in the treatment of acute severe hypertension in children. As outlined, unlike adults with hypertension, in whom administration of shortacting nifedipine may be associated with a higher risk of myocardial infarction, pediatric patients tolerate the drug without evidence of significant irreversible adverse events. It can be used during the transition period when long-acting agents are introduced into the treatment regimen. The drug options for treatment of acute hypertensive emergencies in children are summarized in Table 1. After acutely lowering the blood pressure from the severe levels that are associated with intracranial hemorrhage and other end-organ dysfunction, the patient will need to be started on a regimen of agents to completely normalize the blood pressure.

\section{Chronic hypertension General principles}

In patients with chronic hypertension who require longterm management, it is valuable to build on a foundation of healthy lifestyle choices to maximize efficacy and to reduce end-organ damage related to hypertension. This includes adoption of a healthy, balanced diet, such as the Dietary Approaches to Stop Hypertension (DASH) diet, ${ }^{11}$ weight reduction, regular exercise, smoking cessation, and relaxation techniques, if possible.

In adults, it has been amply documented that even in patients with identified hypertension, only $50 \%$ receive adequate treatment to completely restore normal readings. ${ }^{12}$ It is likely that this therapeutic shortcoming also applies to pediatric patients. Thus, satisfactory control of blood pressure requires careful follow-up and close interaction with the patient and his/her family to ensure that a successful regimen is implemented and that its benefits are sustained.

The choice of blood pressure-lowering agents will be guided by the nature of the underlying condition causing the hypertension and the presence of other comorbid conditions that might be adversely affected by specific drugs.

There are several important principles that need to be followed in prescribing drugs for the long-term control of hypertension in pediatric patients. First, once severe hypertension is brought under control, the patient is unlikely to have any symptoms that are directly due to the elevated blood pressure. Therefore, it is imperative that agents be chosen that do not cause adverse effects on quality of life or undermine the willingness of the patient to maintain the course of treatment. Second, because hypertension in pediatrics is most common in adolescent patients, fostering adherence to treatment represents an integral part of the care of patients in this age cohort. To maximize the likelihood that pediatric patients will actually take their prescribed medications, it is 
Table I Drugs for use in acute hypertension

\begin{tabular}{llll}
\hline Drug & Class & Dose & Side effects \\
\hline Labetalol & $\beta$-blocker & $0.25-1 \mathrm{mg} / \mathrm{kg}$ per hour & Dizziness, nausea, stuffy nose \\
Nicardipine & CCB, intravenous & $0.1-3 \mu g / \mathrm{kg} \mathrm{per} \mathrm{minute}$ & Edema, flushing \\
Enalapril & ACEl & $0.005-0.01 \mathrm{mg} / \mathrm{kg}$ per hour & Facial swelling, cough \\
Diazoxide & Vasodilator & $1 \mathrm{mg} / \mathrm{kg}$ & Hyperglycemia \\
Nitroprusside & Vasodilator & $0.5-2 \mu \mathrm{kg}$ per minute, titrated & Cyanide intoxication \\
Nifedipine & CCB, oral & minute to minute & Edema, flushing \\
\hline
\end{tabular}

Abbreviations: $\mathrm{ACEl}$, angiotensin-converting enzyme inhibitor; $\mathrm{CCB}$, calcium channel blocker.

advisable to use the fewest number of agents possible and to try to prescribe once-daily dosing regimens. Thus, if adding new drugs to a regimen has failed to adequately lower blood pressure, it is advisable to switch to a new drug or to use a combination of drugs that has physiologic justification, such as a diuretic with an ACEI, or a $\beta$-blocker in conjunction with an ACEI. Finally, it is critical to take into account features of normal development, such as growth potential, pubertal status, and cognitive capacity, in designing a therapeutic plan to treat hypertension in pediatric patients. It is advisable to arrange a meeting with the patient prior to starting therapy to explain the rationale for treatment and the justification for the choice of drugs and to explain potential adverse effects, in order to enhance acceptance and adherence by the patient. The drug options for treatment of chronic hypertension in children are summarized in Table 2.

\section{Management of essential hypertension}

As outlined, most pediatric patients with primary hypertension deserve a trial of nonpharmacologic management to lower blood pressure. This applies even though there are preliminary data suggesting that early treatment of prehypertensive adult patients with an angiotensin receptor blocker can delay the onset of sustained hypertension. ${ }^{13}$ A similar study is required in pediatric patients to determine whether intervention when blood pressure readings are classified as prehypertensive can retard or prevent the development of overt hypertension.

In general, children and adolescents are less responsive than adults to salt restriction. ${ }^{14}$ Thus, dietary therapy should focus on the global composition of the diet, with attention given to sodium, potassium, calcium, protein, fruits, and vegetables. Weight loss is difficult to achieve and sustain and usually will require concerted effort by a team of professionals, including a dietician and a nurse. There is evidence that loss of $10-15$ pounds $(4-7 \mathrm{~kg}$ ) is sufficient to achieve a meaningful reduction in blood pressure. This change may be attributable to improved responsiveness to insulin, altered sympathetic nervous system activity, and reversal of endothelial dysfunction. ${ }^{15}$ Regular exercise of any kind, namely participation in physical activities that increase the heart rate for 30-40 minutes three to four times a week, is a realistic goal that leads to a demonstrable improvement in hypertension. ${ }^{16}$ Stress reduction using techniques such as yoga and biofeedback are clearly and highly effective in motivated patients. Nonpharmacologic options for the treatment of hypertension in pediatric patients are summarized in Table 3.

Table 2 Drugs for use in chronic hypertension

\begin{tabular}{llll}
\hline Drug & Class & Dose & Side effects \\
\hline Chlorthalidone & Diuretic & $0.3-2 \mathrm{mg} / \mathrm{kg}$ per day & $\begin{array}{l}\text { Hypokalemia, hyperglycemia, } \\
\text { hyperuricemia, renal injury }\end{array}$ \\
& Maximum: $50 \mathrm{mg}$ & Cough, hyperkalemia, \\
& Given every 24 hours & angioedema, decreased GFR \\
Enalapril & $0.1-0.6 \mathrm{mg} / \mathrm{kg}$ per day & Hyperkalemia, decreased GFR \\
& ARB & Given every I2-24 hours & \\
Losartan & $0.7-1.4 \mathrm{mg} / \mathrm{kg}$ per day & Flushing, edema, headaches \\
& Maximum: $100-150 \mathrm{mg}$ & \\
\end{tabular}

Abbreviations: ACEI, angiotensin-converting enzyme inhibitor; ARB, angiotensin II type I receptor blocker; CCB, calcium channel blocker; GFR, glomerular filtration rate. 
Table 3 Nonpharmacologic options for treatment of pediatric patients with hypertension

\begin{tabular}{l} 
Dietary changes \\
Sodium restriction \\
Potassium supplementation \\
Calcium supplementation \\
Magnesium supplementation \\
Weight loss \\
Exercise \\
Biofeedback \\
Relaxation techniques: yoga, meditation \\
\hline
\end{tabular}

If a reasonable attempt to implement nondrug strategies fails to lower blood pressure in patients with essential hypertension, then initiation of drug therapy is warranted. This approach to therapy is warranted if the child has any symptoms such as headache, if there are other cardiovascular risk factors such as obesity or hyperlipidemia, or if there is a family history of premature cardiovascular morbidity or early deaths from myocardial infarction or cerebrovascular accidents. In the past, efforts were made to individualize therapy based on renin profiling on the ethnicity/race of the patients. Thus, White patients were thought to have primarily reninmediated hypertension, and Black patients were thought to have volume-mediated hypertension, and these assumptions were used to guide the choice of the initial therapeutic agent. In addition, a stepped approach was advised in which second and third agents were added sequentially in a formalized process until blood pressure was normalized. ${ }^{17}$

The results of the ALLHAT (Antihypertensive and Lipid Lowering Treatment to Prevent Heart Attacks Trial) and AASK (African American Study of Kidney Diseases and Hypertension) trials, ${ }^{18,19}$ which enrolled large numbers of White and Black adult patients, respectively, and assessed efficacy of antihypertensive therapy based on the impact on clinically significant endpoints, have significantly altered the way clinicians manage hypertension. ALLHAT compared a CCB (amlodipine), an ACEI (lisinopril), and a thiazide diuretic (chlorthalidone). An $\alpha-1$ adrenoceptor antagonist, doxazosin, ${ }^{20}$ was dropped early in the study because of an increased risk of heart failure. The major conclusion of the trial was that the three drug classes were equivalent in antihypertensive efficacy. Thus, there is no a priori reason to favor one class over the other in White or Black patients who do not have underlying kidney disease. In view of the minimal cost and extended experience with thiazide diuretics, these agents were seen in a new, more favorable light. Although criticism has been directed at this interpretation of ALLHAT, ie, that the CCB and ACEI doses were not maximized and that thoughtful combination therapy was proscribed, the net result has been a resurgence in the use of diuretics as a first-line agent in all patients with hypertension. In fact, although hydrochlorothiazide continues to be the most commonly prescribed diuretic, monitoring of national prescribing trends suggest that there has been a shift toward increased use of chlorthalidone, the drug administered in ALLHAT. ${ }^{21}$ It is clear that there are adverse effects from thiazide diuretics, including hyperglycemia, hyperlipidemia, and hyperuricemia. In addition, in experimental animals, prolonged administration of thiazide diuretics is associated with interstitial fibrosis and tubular atrophy that may be independent of drug-induced hypokalemia. ${ }^{22}$ However, with the recognition that the antihypertensive effect of diuretics requires lower doses than were prescribed in the past, these side effects can be minimized.

There are no comparable studies of the role of diuretics as first-line therapy in pediatric patients with hypertension. However, if anything, in view of the lower incidence of cardiovascular and atherosclerotic heart disease in children versus adults, the overall safety of diuretics should be even greater in pediatric patients compared with in their adult counterparts.

In approximately $50 \%$ of patients with hypertension, the use of diuretics as a single agent will not be adequate to normalize blood pressure. In these cases, a second, third, or even fourth drug may be needed to control hypertension, and updated treatment guidelines by professional societies emphasize this point. ${ }^{23}$ It is important to emphasize that according to data from the National Health and Nutrition Examination Survey (NHANES), many patients with diagnosed hypertension are not provided with sufficient medication to achieve blood pressure in the normal range. ${ }^{12}$ Thus, physicians and nephrologists need to overcome a natural reluctance to prescribe additional medications, provided they are meticulous in trying to utilize thoughtful combinations and once-daily dosing and avoid agents with significant morbidity that will undermine patient adherence to treatment. The use of these additional drugs is covered in the following section. The diagnosis and treatment of essential hypertension in children and adolescents is a costly effort, and recent studies have tried to identify the most cost-effective approach. In general, population-based strategies, such as reduced salt content in manufactured foods and increased exercise in school, yield the greatest benefit for the least cost. Other feasible approaches include screening and treating high-risk patients, such as those who are overweight or who have evidence of target organ injury, eg, left ventricular hypertrophy. ${ }^{24}$ 


\section{Management of secondary hypertension: nonrenal causes}

There is the recognition that regardless of the underlying cause of hypertension, the final common pathway is increased peripheral vascular resistance. This was the justification for the use of direct vasodilators to treat elevated blood pressure levels. In fact, this was the mechanism of action for most of the drugs that were initially used to treat hypertension, eg, ganglionic blockers, hydralazine. However, most of these agents were associated with symptomatic hypotension and a wide range of poorly tolerated side effects. CCBs are the most recently developed direct vasodilators and are widely used precisely because of their generally favorable side effect profile.

The CCBs fall into three distinct molecular categories: dihydropyridines (eg, nifedipine), phenylalkylamines (eg, verapamil), and benzothiazepines (eg, diltiazem). They all share the property of inhibiting calcium entry into the vascular smooth-muscle cell via a voltage-gated channel. Although all three chemical types of CCBs are used in adults, the most widely used drugs in this class are the dihydropyridines. Therefore, there is the greatest experience with these CCBs. These agents are available in short-acting and extended-release formulations. Amlodipine $(0.05-0.5 \mathrm{mg} / \mathrm{kg}$ per day) may be the optimal CCB for use in children because of its enhanced binding to the calcium channel, prolonged elimination half-life ( $>30$ hours), and extended duration of action. ${ }^{25,26}$ This facilitates once-daily dosing for control of hypertension in most cases. Unfortunately, the CCBs are available only as tablets, and a standard liquid preparation has not been designed that is US Food and Drug Administration (FDA) approved for use in children and demonstrated to be bioequivalent to tablets. This manufacturing problem may limit use of CCBs to older children who can swallow tablets.

There are several relatively common side effects of CCBs, including flushing, headaches, migraines, peripheral edema, and congestive heart failure. Less common but important adverse effects include gingival hyperplasia, chest pain, and gastrointestinal complaints such nausea and vomiting.

$\beta$-blockers lower blood pressure via a number of mechanisms, including altered central and peripheral sympathetic nervous system activity, inhibition of renin release, reduction in cardiac output, and potentiation of the action of natriuretic peptides. Agents in this class have been available for over 40 years. However, the initial drugs that were introduced into clinical practice were nonselective and contraindicated in patients with pulmonary disease, because of the risk of bronchoconstriction. The development of cardioselective $\beta$-adrenergic receptor blockers such as atenolol and metoprolol substantially overcame this problem. Labetalol has the additional property of acting as a peripheral $\alpha 1$-adrenergic blocker, which in conjunction with its $\beta$-blocker actions enhances its blood pressure-lowering action. It has become one of the most commonly prescribed $\beta$-blockers in pediatric patients.

$\beta$-blockers can be effective as single agents or in combination with other drugs, such as a diuretic and/or an ACEI. They are metabolized by the liver before entering the systemic circulation, which accounts for wide variability in response to these drugs.

The most common side effects of $\beta$-blockers are related to central nervous system disturbances, including fatigue, depression, dizziness, and lightheadedness. Other adverse events associated with administration of $\beta$-blockers include bradycardia, postural hypotension, cold extremities, and nausea.

Most patients with nonrenal secondary hypertension or primary (essential) hypertension can achieve normal blood pressure using exclusively the agents described previously. As mentioned, ${ }^{23}$ in select cases, additional third and fourth agents may be required. Direct vasodilators such as hydralazine and prazosin can cause reflex tachycardia, palpitations, and symptomatic hypotension, especially after increases in dosage. Hydralazine can trigger a lupus-like illness. Minoxidil, the most potent vasodilator, should be avoided, if possible, because of the induction of hypertrichosis. This is a significant side effect in body image-conscious adolescents. Centrally acting drugs such as clonidine $(0.1-0.9 \mathrm{mg} /$ day in one to three doses) are poorly tolerated because of the associated drowsiness and fatigue. However, it is important to keep this agent in mind in difficult cases, because it is available as a patch that only needs to be applied weekly. This method of transcutaneous drug delivery can improve blood pressure control in adolescents who do not adhere to treatment recommendations, provided there is no abrupt interruption in application of the patch.

\section{Management of secondary hypertension: renal causes}

In patients with underlying renal parenchyma disease, treatment of hypertension accomplishes two distinct purposes: (i) prevention of cardiovascular disease, including myocardial infarction, congestive heart failure, and cerebrovascular events; and (ii) protection against progressive deterioration 
in kidney function. Successful reduction in blood pressure will generally achieve both goals. The target blood pressure for patients with chronic kidney disease (CKD) who have hypertension has been set lower compared with those who have normal kidney function. In adults with CKD, the blood pressure goal is $125-130 / 75-80 \mathrm{~mm} \mathrm{Hg} .{ }^{27} \mathrm{It}$ is worth noting that recent reports, such as the AASK cohort study, have raised questions about the clinical benefit of more intensive control of blood pressure in patients with CKD. However, under these circumstances, there is evidence that use of agents that block the renin-angiotensin axis and the action of aldosterone may have additional benefit with regard to the second objective, namely renoprotection. There are investigators who have argued that there are no blood pressure-independent effects of antagonists/inhibitors of renin-angiotensin on renal function and claim that, provided there is equivalent degree of blood pressure lowering, all classes of antihypertensive drugs are effective in stabilizing renal function in patients with underlying kidney disease. ${ }^{29}$ However, the general consensus is that agents that prevent the synthesis and/or action of angiotensin II and aldosterone have long-term renoprotective effects that exceed the benefits related solely to the reduction in blood pressure. These actions include decreased production of the profibrotic cytokine, transforming growth factor- $\beta$ (TGF- $\beta$ ), reduced activity of proinflammatory molecules, and diminished production of oxygen free radicals. ${ }^{30,31}$ These molecules are mediators of renal injury and fibrosis in patients with $\mathrm{CKD}$, and interference with their action by the administration of agents that inhibit the action of angiotensin II and/or aldosterone have been demonstrated to be beneficial in experimental models of CKD and a wide range of clinical disorders of the glomerulus and tubulointerstitium.

The first class of drugs introduced into clinical practice that interfered with the action of angiotensin II were the ACEIs, eg, captopril, enalapril, and lisinopril, in 1981. They were followed by the antagonists of angiotensin II type 1 receptor blockers (ARBs), eg, losartan, in 1995. The most recently developed class of drugs with the general property of disruption of the renin-angiotensin axis are the direct renin inhibitors, eg, aliskiren. Finally, selective aldosterone antagonists, eg, eplerenone, are now available that do not occupy other steroid receptors or exert an antiandrogen action and thus are devoid of side effects of previous agents in this group. Based on preclinical studies, aldosterone antagonists reduce fibrosis by decreasing the activity of TGF- $\beta$ and plasminogen activator inhibitor- $1 .{ }^{32}$ In the RALES (Randomized Aldactone Evaluation Study), ${ }^{33}$ adding spironolactone to the therapeutic regimen of patients with severe congestive heart failure lowered the incidence of death and cardiovascular complications. In small, uncontrolled clinical trials, administration of eplerenone has been associated with preservation of renal structure and function, to a degree that exceeds expectation based on the degree of blood pressure reduction achieved with their use. These studies require confirmation in large-scale randomized clinical trials.

ACEIs have been part of clinical practice since the early 1980s. They were one of the first custom-designed drugs whose synthesis was based on crystallographic studies of the structure of $\mathrm{ACE}$ and the selection of molecules that would inhibit enzymatic activity. The first drug in this class was captopril, which required dosing two to four times a day and which contained a sulfhydryl group that caused drugrelated toxicity. All currently used ACEIs are prodrugs that are slowly converted to the active form, enabling once-a-day dosing. In addition, they do not contain a sulfhydryl group and are less like to cause neutropenia and loss of taste.

Controlled clinical trials have verified the short-term efficacy of enalapril, lisinopril, ramipril, and fosinopril in the treatment of essential hypertension and secondary hypertension due to CKD in pediatric and adult patients. ${ }^{34,35}$ They are generally successful as a single agent in $60 \%$ of patients (range 6\%-93\%). They rarely cause symptomatic hypertension and are associated with a very favorable impact on quality of life. Their efficacy is enhanced by the addition of a diuretic to the treatment regimen. The diuretic enhances renin release and potentiates the blood pressure-lowering action of ACEIs.

The major clinical side effects of ACEI are cough that occurs in up to $10 \%$ of adults and $3 \%$ of children. ${ }^{36}$ Angioedema is another rare but potentially life-threatening complication that can be treated by discontinuing the ACEI. It rarely recurs after switching to an ARB. ${ }^{37} \mathrm{~A}$ significant number of patients, especially those with $\mathrm{CKD}$, may experience a marked decline in kidney function if they are placed on an ACEI. This is an expected event based on the reduction in intrarenal angiotensin II levels and the decline in intraglomerular perfusion pressure. However, if the fall in glomerular filtration rate (GFR) is $20 \%$ or less compared with the pretreatment value, then the short-term adverse impact is outweighed by the long-term benefit of extended preservation of GFR. ${ }^{38}$ It is worth noting that the renoprotective effects of ACEIs may require larger doses than the amounts needed to maximally lower blood pressure. This may be related to the amount of drug needed to reduce the angiotensin II-mediated changes in inflammatory and profibrotic mediators of CKD 
progression, eg, TGF- $\beta$. In addition, hyperkalemia is a relatively frequent complication of ACEI use, especially in those with CKD who are given these drugs as part of routine clinical care. ${ }^{39}$ Thus, this group of patients requires close monitoring of kidney function and serum electrolyte concentrations when an ACEI is added to the therapeutic regimen. ${ }^{40}$ Finally, female patients of child-bearing age must be warned about the potential teratogenic effects of ACEIs that are applicable throughout gestation. ${ }^{41}$

ARBs represent a viable option in patients whose blood pressure cannot be controlled with an ACEI or who develop adverse events in response to the latter class of agents. They may have additional benefits compared with the ACEIs because they would inhibit the action of angiotensin II that is synthesized by non-ACE-dependent pathways, eg, chymase. ${ }^{42}$ There is evidence from animals that the beneficial effect of ARBs is related to two parallel pharmacologic actions: blockade of the vasoconstrictor angiotensin type 1 (AT1) receptor and diversion of angiotensin II to the angiotensin type 2 (AT2) receptor. Occupancy of the latter receptor increases synthesis of nitric oxide, leading to vasodilatation and lowering of blood pressure. ${ }^{42}$

These agents have been demonstrated to be effective in lowering blood pressure in pediatric and adult patients. The overall side effect profile is similar to that of ACEIs. However, because they are not associated with inhibition of bradykinin degradation, a direct action of ACE, they have a lower likelihood of triggering a cough. ${ }^{36}$

Currently, there is ongoing debate about whether combined use of an ACEI and an ARB yields any benefit on blood pressure control and protection against progressive decline in renal function compared with maximal use of either drug as a single agent. ${ }^{43,44}$ There is evidence to support both positions. The consensus on this practice point is to maximize the dose of each agent individually before adding a drug from the other class. In addition, patients prescribed both agents in combination require close surveillance for the development of hyperkalemia or a decline in GFR. ${ }^{39,45}$

One predictable response to the use of an ACEI or an ARB is an increase in the plasma renin activity (PRA) secondary to loss of feedback inhibition by angiotensin II. This compensatory rise in PRA may overcome the pharmacologic blockade of ACE or the AT1 receptor and account for the gradual loss of activity of these drugs. This has spurred the development of agents that directly affect the action of renin on angiotensinogen to form angiotensin I. ${ }^{45,46}$ Interestingly, part of the beneficial effect of aliskiren may be attributable to inhibition of the prorenin receptor, which may directly activate profibrotic signaling pathways within the kidney independently of the AT1 receptor. ${ }^{47}$ The first drug in this class that has been FDA approved and introduced into clinical use is aliskiren. It is available in tablet form and has been shown to achieve a reduction in blood pressure that is comparable with ACEIs or ARBs. The long-term effects on blood pressure and renal function will be determined with prolonged experience with this agent. There is considerable controversy on whether the CCBs exert a renoprotective effect that is greater than the drug-induced fall in blood pressure, and it is for that reason that drugs that target reninangiotensin II are still given preference in patients with underlying CKD and proteinuria. ${ }^{48,49}$

In most patients with renal disease, additional drugs to agents that interrupt the renin-angiotensin axis are needed to control blood pressure. These are outlined in the previous section, which describes drug therapy in patients who are hypertensive but who do not have kidney disease.

\section{Conclusion}

Hypertension is an increasingly prevalent problem in pediatrics. Therefore, both general pediatricians and specialists need to be familiar with the therapeutic approach to treating this problem. Although nonpharmacologic therapy is preferred, it is often inadequate to normalize high blood pressure, and drug treatment is necessary. The goal of treatment is different in the setting of acute life-threatening hypertension and sustained elevations in blood pressure due to an underlying disease. Regardless of the underlying cause, a regimen should be selected that addresses the specific clinical circumstances, minimizes side effects to maximize adherence, and has the least long-term toxicity. Further research is likely to lead to identification of additional therapeutic targets that will allow the implementation of personalized medicine for the treatment of hypertension. Drug development will foster the manufacture of safer agents with a higher benefit:risk ratio, which, in turn, will alleviate some of the persistent concerns related to prolonged therapy of hypertension in pediatric patients.

\section{Disclosure}

The author has no conflicts of interest to disclose in relation to this work.

\section{References}

1. Muntner P, He J, Cutler JA, et al. Trends in blood pressure among children and adolescents. JAMA. 2004;291:2107-2213.

2. Wiesen J, Adkins M, Fortune S, et al. Evaluation of pediatric patients with mild-to-moderate hypertension: yield of diagnostic testing. Pediatrics. 2008;122:e993-e998. 
3. Urbina E, Alpert B, Flynn J, et al. Ambulatory blood pressure monitoring in children and adolescents: recommendations for standard assessment: a scientific statement from the American Heart Association, and Obesity in Youth Committee of the Council on Cardiovascular Disease in the Young and the Council for High Blood Pressure Research. Hypertension. 2008:52:433-451.

4. Flynn JT. Pediatric hypertension update. Curr Opin Nephrol Hypertens. 2010;19:292-297.

5. National high blood pressure education program working group on high blood pressure in children and adolescents. The fourth report on the diagnosis, evaluation, and treatment of high blood pressure in children and adolescents. Pediatrics. 2004;114;555-576.

6. Proulx F, Lacroix J, Farrell CA, Gauthier M. Convulsions and hypertension in children: differentiating cause from effect. Crit Care Med 1993;21:1541-1546.

7. Calhoun DA, Oparil S. Treatment of hypertensive crisis. N Engl J Med. 1990;323:1177-1183.

8. Miyashita Y, Peterson D, Rees JM, Flynn JT. Isradipine for treatment of acute hypertension in hospitalized patients and adolescents. $J$ Clin Hypertens. 2010;12:850-855.

9. Yiu V, Orrbine E, Rosychuk RJ, et al. The safety and use of short-acting nifedipine in hospitalized hypertensive children. Pediatr Nephrol. 2004;19:644-650.

10. van Harten J, Burggraff K, Danhof M, et al. Negligible sublingual absorption of nifedipine. Lancet. 1987;2:1363-1365.

11. Sacks FM, Svelkey LP, Vollmer WM, et al. Effects on blood pressure of reduced dietary sodium and the Dietary Approaches to Stop Hypertension (DASH) diet. DASH Sodium Collaborative Research Group N Engl J Med. 2001;344:3-10.

12. Egan BM, Zhao Y, Axon RN. US trends in prevalence, awareness, treatment, and control of hypertension, 1988-2008. JAMA. 2010;303: 2043-2050.

13. Julius S, Nesbitt SD, Egan BM, et al. Feasibility of treating prehypertension with an angiotensin receptor blocker. N Engl J Med. 2006;354: 1685-1697.

14. Couch SC, Daniels SR. Diet and blood pressure in children. Curr Opin Pediatr. 2005;17;642-647.

15. Kotchen TA. Obesity-related hypertension. Am J Hypertens. 2010;23: 1170-1178.

16. Blumenthal JA, Babyak MA, Sherwood A, et al. effects of the dietary approaches to stop hypertension diet alone in combination with exercise and caloric restriction on insulin sensitivity and lipids. Hypertension. 2010;55:1199-1205

17. Chobanian AV, Bakris GL, Black HR, et al. Seventh report of the Joint National Committee on Prevention, Detection, Evaluation, and Treatment of High Blood Pressure. Hypertension. 2003;42:1206-1252.

18. Wright JT Jr, Dunn JK, Cutler JA, et al. Outcomes of hypertensive black and nonblack patients treated with chlorthalidone, amlodipine, and lisinopril. JAMA. 2005;293;1595-1608.

19. Wright JT Jr, Bakris G, Greene T, et al. effect of blood pressure lowering and antihypertensive drug class on progression of hypertensive kidney disease: results from the AASK trial. JAMA. 2002;288:2421-2431.

20. Chapman N, Chen CY, Fujita T, et al. Time to re-appraise the role of alpha-1 adrenoceptor antagonists in the management of hypertension? J Hypertens. 2010;28:1796-1803.

21. Ernst ME, Lund BC. Renewed interest in chlorthalidone: evidence from the Veterans Health Administration. J Clin Hypertens. 2010;12: 927-934.

22. Reungjui $\mathrm{S}, \mathrm{Hu} \mathrm{H}, \mathrm{Mu} \mathrm{W}$, et al. Thiazide-induced subtle renal injury not observed in states of equivalent hypokalemia. Kidney Int. 2007;72: 1483-1492.

23. Flack JM, Sica DA, Bakris G, et al. Management of high blood pressure in Blacks: an update of the International Society of Hypertension in Blacks consensus statement. Hypertension. 2010;56:780-800.

24. Wang YC, Cheung AM, Bibbins-Domingo K, et al. Effectiveness and cost-effectiveness of blood pressure screening in adolescents in the United States. J Pediatr. 2011;158:257-264.
25. Flynn JT, Newburger JW, Daniels SR, et al. A randomized, placebocontrolled trial of amlodipine in children with hypertension. J Pediatr. 2004;145:353-359.

26. Flynn JT, Nahata MC, Mahan JD Jr, Portman RJ. Population pharmacokinetics of amlodipine in hypertensive children and adolescents. J Clin Pharmacol. 2006;46:905-916.

27. The ESCAPE Trial Group. Strict blood-pressure control and progression of renal failure in children. $N$ Engl J Med. 2009;361:1639-1650.

28. Appel LJ, Wright JT Jr, Greene T, et al. Intensive blood-pressure control in hypertensive chronic kidney disease. N Engl J Med. 2010;363: 918-929.

29. Bidani AK, Griffin KA. Pathophysiology of hypertensive renal damage: implications for therapy. Hypertension. 2004;44:595-601.

30. Ruggenenti P. Angiotensin-converting enzyme inhibition and angiotensin II antagonism in nondiabetic chronic nephropathies. Semin Nephrol. 2004;24:158-167.

31. Hou FF, Zhou QG. Optimal dose of angiotensin-converting enzyme inhibitor or angiotensin II receptor blocker for renoprotection. Nephrology. 2010;15(Suppl 2):57-60.

32. Ma J, Weisberg A, Griffin JP, et al. Plasminogen activator inhibitor-1 deficiency protects against aldosterone-induced glomerular injury. Kidney Int. 2006;69:1064-1072.

33. Pitt B, Zannad F, Remme WJ, Cody R, et al. the effect of spironolactone on morbidity and mortality in patients with severe heart failure. Randomized Aldactone Evaluation Study. N Engl J Med. 1999;341: 709-717.

34. Wells T, Frame V, Soffer B, et al. A double-blind, placebo-controlled, dose-response study of the effectiveness and safety of enalapril for children with hypertension. J Clin Pharmacol. 2002;42:870-880.

35. Wuhl E, Mehls O, Schaeffer F. Antihypertensive and antiproteinuric efficacy of ramipril in children with chronic renal failure. Kidney Int. 2004;66:768-776.

36. Baker-Smith CNM, Benjamin DK Jr, Califf RM, et al. Cough in pediatric patients receiving angiotensin-converting enzyme inhibitor therapy or angiotensin receptor blocker therapy in randomized clinical trial. Clin Pharm Therap. 2010;87:668-671.

37. Cicardi M, Zingale LC, Bergamaschini L, Agostoni A. Angioedema associated with angiotensin-converting enzyme inhibitor use. Arch Int Med. 2004;164:910-913.

38. Palmer BF. Renal dysfunction complicating treatment of hypertension. N Eng J Med. 2002;347:1256-1261.

39. Bakris GL, Weir MR. Comparison of dual RAAS blockade and higherdose RAAS inhibition on nephropathy progression. Postgrad Med. 2008;1210:33-42.

40. Palmer BF. Managing hyperkalemia caused by inhibitors of the reninangiotensin-aldosterone system. $N$ Eng J Med. 2004;351:585-592.

41. Cooper WO, Hernandez-Diaz S, Arbogast PG, et al. Major congenital malformations after first trimester exposure to ACE inhibitors. $N$ Engl J Med. 2006;354:2443-2451.

42. Petrie MC, Padmanabhan N, McDonald JE, et al. Angiotensin converting enzyme (ACE) and non-ACE dependent angiotensin II generation in resistance arteries from patients with heart failure and coronary heart disease. J Am Coll Cardiol. 2001;37:1056-1061.

43. Hirata K, Vlachopoulosa C, Adji A, O'Rourke MF. Benefits from angiotensin converting enzyme inhibitor "beyond blood pressure lowering": beyond blood pressure or beyond the brachial artery? J Hypertens. 2005;23:551-556.

44. Matchar DB, McCrory DC, Orlando LA, et al. Systematic review: comparative effectiveness of angiotensin-converting enzyme inhibitors and angiotensin II receptor blockers for treatment of essential hypertension. Ann Int Med. 2008;148:16-29.

45. Norris S, Weinstein J, Peterson K, Thakurta S. Direct renin inhibitors, angiotensin converting enzyme inhibitors, and angiotensin II receptor blockers: final report. Drug Class Reviews. Portland, OR: Oregon Health and Science University; 2010.

46. Mende CW. Application of direct renin inhibition to chronic kidney disease. Cardiovasc Drug Ther. 2010;24:139-149. 
47. Gross O, Girgert R, Rubel D, et al. Renal protective effects of aliskiren beyond its antihypertensive property in a mouse model of progressive fibrosis. Am J Hypertens. 2011;24(3):355-361.

48. Nathan S, Pepine CJ, Bakris GL. Calcium antagonists: effects on cardiorenal risk in hypertensive patients. Hypertension. 2005;46:637-642.
49. Sarafidis PA, Khosia N, Bakris GL. Antihypertensive therapy in the presence of proteinuria. Am J Kid Dis. 2007;49:12-26.

Integrated Blood Pressure Control

Dovepress

\section{Publish your work in this journal}

Integrated Blood Pressure Control is an international, peer-reviewed open-access journal focusing on the integrated approach to managing hypertension and risk reduction. Treating the patient and comorbidities together with diet and lifestyle modification and optimizing healthcare resources through a multidisciplinary team approach constitute key Society's Chemical Abstracts Service (CAS). The manuscript management system is completely online and includes a very quick and fair peerreview system, which is all easy to use. Visit http://www.dovepress.com/ testimonials.php to read real quotes from published authors.

Submit your manuscript here: http://www.dovepress.com/integrated-blood-pressure-control-journal 